\title{
Authenticated Routing for Ad hoc Networks
}

\author{
Kimaya Sanzgiri* \\ Daniel LaFlamme ${ }^{\dagger}$ \\ Bridget Dahill ${ }^{\dagger}$ \\ Brian Neil Levine ${ }^{\dagger}$ \\ Clay Shields ${ }^{\ddagger}$ \\ Elizabeth M. Belding-Royer* \\ * Dept. of Computer Science, University of California, Santa Barbara, CA 93106 \\ ${ }^{\dagger}$ Dept. of Computer Science, University of Massachusetts, Amherst, MA 01060 \\ $\ddagger$ Dept. of Computer Science, Georgetown University, Washington, DC 20057
}

\begin{abstract}
Initial work in ad hoc routing has considered only the problem of providing efficient mechanisms for finding paths in very dynamic networks, without considering security. Because of this, there are a number of attacks that can be used to manipulate the routing in an ad hoc network. In this paper, we describe these threats, specifically showing their effects on AODV and DSR. Our protocol, named Authenticated Routing for Ad hoc Networks (ARAN), uses public-key cryptographic mechanisms to defeat all identified attacks. We detail how ARAN can secure routing in environments where nodes are authorized to participate but untrusted to cooperate, as well as environments where participants do not need to be authorized to participate. Through both simulation and experimentation with our publicly-available implementation, we characterize and evaluate ARAN and show that it is able to effectively and efficiently discover secure routes within an ad hoc network.
\end{abstract}

\section{INTRODUCTION}

Securing protocols for mobile ad hoc networks presents unique challenges due to characteristics such as lack of predeployed infrastructure, centralized policy and control. In this paper, we make a number of contributions to the design of secure ad hoc routing protocols ${ }^{1}$. First, we describe exploits that are possible against ad hoc routing protocols. We show specifically that two protocols that are under consideration by the IETF for standardization, AODV [9] and DSR [10], although efficient in terms of network performance, are replete with security flaws.

Second, we define and distinguish the heterogeneous environments that make use of ad hoc routing and differ in their assumed pre-deployment and security requirements. This approach is important because satisfying a tighter set of security requirements than an application requires is unwarranted and wasteful of resources.

Third, we propose a secure routing protocol, Authenticated Routing for Ad hoc Networks (ARAN), that detects and protects against malicious actions by third parties and peers. ARAN introduces authentication, message integrity, and nonrepudiation to routing in an ad hoc environment as a part of a minimal security policy.

We detail how ARAN can be used in two environments: where mobile users are federated and can be pre-certified (e.g.,

Supported in part by National Science Foundation awards ANI-522564, ANI-0335302 and EIA-0080199, by an AFOSR MURI grant, and by U.S. Dept. of Justice, Office of Justice Programs grant 2000-DT-CX-K001. Contents are solely the responsibility of the authors and do not necessarily represent the official views of the DoJ or NSF.

${ }^{1}$ This paper represents many refinements and extensions to our original work from IEEE ICNP 2002 [8]. on a campus) though remain untrusted; and where they are unknown to each other and cannot be pre-certified (e.g., a "rooftop" access point). To our knowledge, ARAN is the first proposal for securing ad hoc routing for rooftop networks.

We analyze the security of ARAN and evaluate its network performance through measurement of both our publiclyavailable implementation and extensive simulations. We find that although there is a greater performance cost to ARAN as compared to DSR or AODV, the increase in cost is minimal and outweighed by the increased security.

This paper is organized as follows. Section II presents an overview of recent work on ad hoc network security. Section III describes the security exploits possible in ad hoc routing protocols. Three ad hoc environments and the security requirements of any ad hoc network are defined in Section IV. Section V presents our secure ad hoc routing protocol, ARAN. A security analysis of ARAN is provided in Section VI, while section VII evaluates ARAN through implementation and simulations. Finally, section VIII offers concluding remarks.

\section{BACKGROUND}

Several proposed ad hoc routing protocols, for example [9], [10], [11], [12], [13], have security vulnerabilities and exposures that easily allow for routing attacks. While these vulnerabilities are common to many protocols, in this paper we focus on two protocols that are under consideration by the IETF for standardization: AODV [9] and DSR [10].

The fundamental differences between ad hoc networks and standard IP networks necessitate the development of new security services. This point has been recognized, and several researchers have examined security problems in ad hoc networks. Numerous solutions have been proposed for providing a secure and reliable certification authority in ad hoc networks [14], [15], [16], [17]. Another problem that has received attention is that of stimulating cooperation among nodes in an ad hoc network and addressing malicious packet dropping [18], [19], [20], [21], [22], [23]. Strategies used include detecting and punishing non-cooperating nodes, rewarding nodes for forwarding packets, concealing the true destination of packets from intermediate nodes, and using redundant data transmissions over multiple paths.

The issue of secure routing in particular has received significant attention. Hu et al. have proposed Ariadne [6], a secure version of DSR. Ariadne can use pre-deployed pairwise symmetric keys or pre-deployed asymmetric cryptography for 
authentication. The former is more efficient, but requires shared secrets between communicating nodes, which may not always be feasible to establish. A third option for Ariadne is the TESLA authentication scheme, which is also based on asymmetric encryption, thus requiring a certification authority or pre-deployed keys. TESLA requires that packets are delayed by the longest RTT in the network before they are sent (thus route creation incurs this delay in both request and response phases).

Chu et al. developed a secure proactive routing protocol based on DSDV [13] called SEAD [24], which is also based on public-key signed hash chains.

SAODV [25], an early attempt to secure the AODV routing protocol, has numerous security vulnerabilities. For instance, it allows a malicious intermediate node to spoof its identity, illegally modify the hop count on route request messages, and fabricate route error messages.

The use of security parameters, such as the trust level of a node in a hierarchical organization, as a routing metric is proposed in [26]. To secure the scheme, the authors suggest that all nodes at the same level of trust should share a common secret. This is not very practical, and has many keymanagement issues.

In an alternative scheme, Papadimitratos et al. [27] propose the Secure Routing Protocol (SRP); however, this is vulnerable to attacks such as fabricated route error messages. Routing security in sensor networks has been analyzed in [28].

The wormhole attack against secure ad hoc routing protocols is studied and a solution is presented in [29], though implementing the solution requires specialized hardware to achieve a high degree of clock synchronization. Awerbuch et al. design a flooding-free reactive routing protocol based on Swarm Intelligence and the Distributed Reinforcement Learning paradigm [30], which is secure against a dynamic Byzantine adversarial model. Finally, intrusion detection techniques for ad hoc networks have been studied [31], [32].

Our work differs from other work in that we do not assume any hardware modifications or synchronized clocks, and only minimal advance keying from a trusted authority. We also account for the costs of distributing cryptographic material instead of assuming it is pre-deployed.

In comparison against related work (e.g.,[6], [7]), ARAN has higher computational costs at each node, which has implications for power costs and latency. However, the dominant energy cost of wireless networking on handheld devices is the idle system with an idle radio [3]; the costs of ARAN's cryptography represent a small price in comparison. ARAN's computational delays are comparable to the mandatory authentication delays required by TESLA [7], a hash-chain-based approach to security. TESLA mandates delays equal to twice the diameter RTT of the network in addition to processing delays, even if the path is between direct neighbors.

\section{Exploits AgAinst Existing PROTOCOLS}

Several popular ad hoc routing protocols allow for many different types of attacks. In this section, we classify and briefly

\begin{tabular}{|c|c|c|c|}
\hline Attack & AODV & DSR & ARAN \\
\hline \multirow{5}{*}{$\begin{array}{l}\text { Remote redirection } \\
\text { modif. of seq. numbers } \\
\text { modif. of hop counts } \\
\text { modif. of source routes } \\
\text { tunneling }\end{array}$} & & & \\
\hline & Yes & No & No \\
\hline & Yes & No & No \\
\hline & No & Yes & No \\
\hline & Yes & Yes & $\begin{array}{l}\text { Yes, but only } \\
\text { to lengthen } \\
\text { path }\end{array}$ \\
\hline Spoofing & Yes & Yes & No \\
\hline \multirow{2}{*}{$\begin{array}{l}\text { Fabrication } \\
\text { fabr. of error messages }\end{array}$} & & & \\
\hline & Yes & Yes & $\begin{array}{l}\text { Yes, but non- } \\
\text { repudiable }\end{array}$ \\
\hline $\begin{array}{l}\text { fabr. of source routes } \\
\text { (cache poisoning) }\end{array}$ & No & Yes & No \\
\hline
\end{tabular}

TABLE I

VULNERABILITIES OF AODV, DSR, AND ARAN.

describe modification, impersonation, and fabrication exploits against ad hoc routing protocols. Detailed descriptions of the attacks can be found in our previous work [8]. In addition, several attacks are possible in the forwarding operation. Data packets can be dropped, replayed, or redirected. In Section V, we propose a protocol that is not exploitable in these ways.

Our focus is on vulnerabilities and exposures that result from the specification of the ad hoc routing protocol, and not from problems with IEEE 802.11. Additionally, denial-ofservice attacks based on non-cooperation and packet dropping, or resource depletion by aggressive route request flooding, are possible in all ad hoc routing protocols. We do not deal with the issue of ensuring protocol compliance, and look only at security problems arising from manipulation of the network routing.

The attacks presented below are described in terms of the AODV and DSR protocols, which we use as representatives of ad hoc on-demand protocols. Table I provides a summary of each protocol's vulnerability to the following exploits.

\section{A. Attacks Using Modification}

Malicious nodes can cause redirection of network traffic and DoS attacks by altering control message fields or by forwarding routing messages with falsified values. Below we briefly describe several modification attacks against AODV and DSR.

1) Redirection by Modified Route Sequence Numbers: Protocols such as AODV and DSDV assign monotonically increasing sequence numbers to routes towards specific destinations. A route with a higher sequence number is preferred over one with a lower sequence number. Thus, in AODV, any node may divert traffic through itself by advertising a route to a node with a destination_sequence_num greater than the authentic value.

2) Redirection with Modified Hop Counts: In AODV, a redirection attack is possible by modification of the hop count field in route discovery messages. When routing decisions cannot be made by other metrics, AODV uses the hop count field to determine a shortest path. Malicious nodes can increase the chances they are included on a newly created route by 
resetting the hop count field of the RREQ to zero. Similarly, by setting the hop count field of the RREQ to infinity, created routes will tend to not include the malicious node. Such an attack is most threatening when combined with spoofing, described in Section III-B.

3) Denial-of-service with Modified Source Routes: DSR utilizes source routes, thereby explicitly stating routes in data packets. These routes lack any integrity checks and a simple denial-of-service attack can be launched in DSR by altering the source routes in packet headers, such that the packet can no longer be delivered to the destination.

4) Tunneling: Ad hoc networks have an implicit assumption that any node can be located adjacent to any other node. A tunneling attack is where two or more nodes collaborate to encapsulate and exchange messages along existing data paths. Such collaborating nodes can pretend to be neighbors, and falsely represent the length of available paths by preventing honest intermediate nodes from correctly incrementing the path length metric.

It is also possible that instead of tunneling through existing multi-hop routes, the malicious nodes can use a long-range directional wireless link or a wired link between them. Such a link gives the attackers an unfair advantage towards occurring on the shortest delay route between a source and destination. This has been referred to as the wormhole attack in recent literature [6], [29]. However, if the malicious nodes truly lie on the shortest delay path, it could be argued that the selection of this path is not a subversion of the routing protocol. A mechanism for defending against wormhole attacks is presented in [29].

\section{B. Attacks Using Impersonation}

Spoofing occurs when a node misrepresents its identity in the network, such as by altering its MAC or IP address in outgoing packets, and is readily combined with other attacks, such as those based on modification. The advantage of spoofing is that the attack cannot be traced back to the malicious node.

\section{Attacks Using Fabrication}

Fabrication attacks involve the generation of false routing messages. Such attacks can be difficult to verify as invalid constructs, especially in the case of fabricated error messages that claim a neighbor cannot be contacted.

1) Falsifying Route Errors in AODV and DSR: In AODV and DSR, if the destination node or an intermediate node along an active path moves, the node upstream of the link break broadcasts a route error message to all active upstream neighbors. This message causes the corresponding route to be invalidated in all upstream nodes. A denial-of-service attack can be launched by continually sending route error messages indicating a broken link on the route, thereby preventing the source from communicating with the destination.

2) Route Cache Poisoning in DSR: In DSR, a node overhearing any packet may add the routing information contained in that packet's header to its own route cache, even if that node is not on the path from source to destination. An attacker could easily exploit this method of learning routes and poison route caches by transmitting packets containing invalid routes in their headers.

\section{SeCURity Requirements of AD HOC NETWORKS}

Applications for ad hoc networks include military operations, emergency rescue missions, and simple provisioning of wireless network access, such as at a conference or in a classroom. In this section, we classify ad hoc networks into three distinct environments that differ in security needs and assumed pre-deployment. These classes are defined because it is difficult to construct a single secure ad hoc routing protocol to suit the needs of many heterogeneous wireless applications. The lower security requirements of some environments do not justify use of costly protocols that satisfy stricter security policies. The environments defined in this section enable us to clearly state where we expect to apply our secure protocol.

A good secure routing algorithm prevents each of the exploits presented in Section III; it must ensure that no node can prevent successful route discovery and maintenance between any other nodes other than by non-participation.

We define a set of three discrete ad hoc wireless environments: open, managed-open, and managed-hostile. These differ not only in the level of security needed, but also in that some have opportunity for exchange of security parameters before the nodes are deployed.

In sum, all secure ad hoc routing protocols must satisfy the following requirements to ensure that path discovery from source to destination functions correctly in the presence of malicious adversaries: (1) Route signaling cannot be spoofed; (2) Fabricated routing messages cannot be injected into the network; (3) Routing messages cannot be altered in transit, except according to the normal functionality of the routing protocol; (4) Routing loops cannot be formed through malicious action; (5) Routes cannot be redirected from the shortest path by malicious action.

These requirements help define an open environment along with the following distinction: all nodes can be considered authorized. This scenario might exist, for example, for a user walking through an urban environment or driving on a highway.

Managed-open environments are accordingly distinguished by an additional requirement: (6) Unauthorized nodes must be excluded from route computation and discovery. This requirement does not preclude the fact that authenticated peers may act maliciously as well. Additionally, we assume that the managed-open environment has the opportunity for predeployment or exchange of public keys, session keys, or certificates. We expect mobile nodes in this environment reside within some common context or geographic proximity. Such an ad hoc network might be formed by peers at a conference, or students on a campus.

We define a managed-hostile environment to have requirements listed above as well as the following: (7) The network topology must neither be exposed to adversaries nor to authorized nodes by the routing messages. A managed-hostile environment is formed, for example, by military nodes in a 


\begin{tabular}{|l|l|}
\hline \hline$K_{A+}$ & Public key of node $A$. \\
$K_{A-}$ & Private key of node $A$. \\
$K_{A B}$ & Symmetric key shared by nodes $A$ and $B$. \\
$\{d\} K_{A+}$ & Encryption of data $d$ with key $K_{A+}$ \\
{$[d] K_{A-}$} & Data $d$ digitally signed by node $A$. \\
cert $_{A}$ & Certificate belonging to node $A$. \\
$e$ & Certificate expiration time. \\
$N_{A}$ & Nonce issued by node $A$. \\
$\mathrm{IP}_{A}$ & IP address of node $A$. \\
$\mathrm{RDP}$ & Route Discovery Packet identifier. \\
$\mathrm{REP}$ & REPly packet identifier. \\
$t$ & timestamp. \\
\hline \hline
\end{tabular}

TABLE II

TABLE OF VARIABLES AND NOTATION.

battle environment, or perhaps by emergency response crews in a disaster area. In such an environment, nodes are deployed by a common source. Consequently, there may be opportunity for pre-deployed exchange of security parameters. The distinguishing security threat of the managed-hostile environment is that every node is vulnerable to physical capture and take-over of equipment, where hostile entities can then pose as friendly entities at a compromised node. Therefore, exposure of node location from the routing protocol messages is not desirable, else adversaries may gain an opportunity to annihilate users.

In the next section we present the ARAN protocol, which meets the needs of the managed-open and open environments. It does not provide a solution to the managed-hostile environment because it exposes the routing topology.

\section{Authenticated Routing For AD HOC NETWORKS}

In this section, we detail the operation of ARAN. ARAN uses cryptographic certificates to prevent most of the attacks presented in Section III and detect erratic behavior.

ARAN consists of a preliminary certification process followed by a route instantiation process that guarantees endto-end authentication. The protocol is simple compared to most non-secured ad hoc routing protocols, and does not include routing optimizations present in the latter. It should be noted that these optimizations are the chief cause of most exploits listed in Section III. Route discovery in ARAN is accomplished by a broadcast route discovery message from a source node that is replied to by the destination node. The routing messages are authenticated end-to-end and only authorized nodes participate at each hop between source and destination.

\section{A. Certification of Authorized Nodes}

ARAN uses cryptographic certificates to bring authentication, message-integrity and non-repudiation to the route discovery process. ARAN therefore requires the use of a trusted certificate server $T$, whose public key is known to all valid nodes (or multiple servers may be used [17]). Nodes use these certificates to authenticate themselves to other nodes during the exchange of routing messages. The use of public keys and certificates is common in many secure ad hoc routing protocols, but most assume the existence of such information without any explicit description of how it is transmitted. While ARAN may appear more expensive, it is in part because we account for the distribution of the cryptographic keying material.

In managed-open environments, keys are a priori generated and exchanged through an existing, perhaps out-of-band, relationship between $T$ and each node. Before entering the ad hoc network, each node must request a certificate from $T$. Each node receives exactly one certificate after securely authenticating its identity to $T$. Details of how certificates are revoked are explained below in Section V-G. Section V-H describes the certification process for open environments.

A node $A$ receives a certificate from $T$ as follows:

$$
T \rightarrow A: \operatorname{cert}_{A}=\left[I P_{A}, K_{A+}, t, e\right] K_{T-}
$$

The certificate contains the IP address of $A\left(\operatorname{IP}_{A}\right)$, the public key of $A\left(\mathrm{~K}_{A+}\right)$, a timestamp $t$ of when the certificate was created, and a time $e$ at which the certificate expires. Table II summarizes our notation. These variables are concatenated and signed by $T$. All nodes must maintain fresh certificates with the trusted server.

\section{B. Authenticated Route Discovery}

The goal of end-to-end authentication is for the source to verify that the intended destination was reached. The source trusts the destination to select the return path.

The source node, $A$, begins route instantiation to destination $X$ by broadcasting to its neighbors a route discovery packet (RDP):

$$
A \rightarrow \text { broadcast : }\left[\mathrm{RDP}, \mathrm{IP}_{X}, N_{A}\right] K_{A-}, \operatorname{cert}_{A}
$$

The RDP includes a packet type identifier ("RDP”), the IP address of the destination $\left(\mathrm{IP}_{X}\right), A$ 's certificate $\left(\operatorname{cert}_{A}\right)$ and a nonce $N_{A}$, all signed with $A$ 's private key. Note that the RDP is only signed by the source and not encrypted, so the contents can be viewed publicly. The purpose of the nonce is to uniquely identify an RDP coming from a source. Each time $A$ performs route discovery, it monotonically increases the nonce. The nonce is 5 bytes in size, and is thus large enough that it will not need to be recycled within the lifetime of the network. ${ }^{2}$ Note that a hop count is not included with the message.

When a node receives an RDP message, it sets up a reverse path back to the source by recording the neighbor from which it received the RDP. This is in anticipation of eventually receiving a reply message that it will need to forward back to the source. The receiving node uses $A$ 's public key, which it extracts from $A$ 's certificate, to validate the signature and verify that $A$ 's certificate has not expired. The receiving node also checks the $\left(N_{A}, \mathrm{IP}_{A}\right)$ tuple to verify that it has not already processed this RDP; nodes do not forward messages with already-seen tuples. The receiving node signs the contents of the message, appends its own certificate, and forward

\footnotetext{
${ }^{2}$ If a source sends a new RDP every millisecond, with a 5 byte nonce, it would take more than 34 years for the value to wrap around.
} 
broadcasts the message to each of its neighbors. The signature prevents spoofing attacks that may alter the route or form loops.

Let $B$ be a neighbor that has received from $A$ the RDP broadcast, which it subsequently rebroadcasts.

$$
B \rightarrow \text { broadcast : }\left[\left[\mathrm{RDP}, \mathrm{IP}_{X}, N_{A}\right] K_{A-}\right] K_{B-}, \operatorname{cert}_{A}, \operatorname{cert}_{B}
$$

Upon receiving the RDP, $B$ 's neighbor $C$ validates the signatures for both $A$, the RDP initiator, and $B$, the neighbor it received the RDP from, using the certificates in the RDP. $C$ then removes $B$ 's certificate and signature, records $B$ as its predecessor, signs the contents of the message originally broadcast by $A$ and appends its own certificate. $C$ then rebroadcasts the RDP.

$$
\left.\left.C \rightarrow \text { broadcast : [[RDP, } \mathrm{IP}_{X}, N_{A}\right] K_{A-}\right] K_{C-}, \operatorname{cert}_{A}, \operatorname{cert}_{C}
$$

Each intermediate node along the path repeats the same steps as $C$.

\section{Authenticated Route Setup}

Eventually, the message is received by the destination, $X$, who replies to the first RDP that it receives for a source and a given nonce. This RDP need not have traveled along the path with the least number of hops; the least-hop path may have a higher delay, either legitimately or maliciously manifested. In this case, however, a non-congested, non-least-hop path is likely to be preferred to a congested least-hop path because of the reduction in delay. Because RDPs do not contain a hop count or specific recorded source route, and because messages are signed at each hop, malicious nodes have no opportunity to redirect traffic with the exploits we described in Section III.

After receiving the RDP, the destination unicasts a Reply (REP) packet back along the reverse path to the source. Let the first node that receives the REP sent by $X$ be node $D$.

$$
X \rightarrow D:\left[\operatorname{REP}, \mathrm{IP}_{a}, N_{A}\right] K_{X-}, \operatorname{cert}_{x}
$$

The REP includes a packet type identifier ("REP"), the IP address of $A\left(\mathrm{IP}_{a}\right)$, the certificate belonging to $X\left(\operatorname{cert}_{x}\right)$ and the nonce sent by $A$. Nodes that receive the REP forward the packet back to the predecessor from which they received the original RDP. Each node along the reverse path back to the source signs the REP and appends its own certificate before forwarding the REP to the next hop. Let $D$ 's next hop to the source be node $C$.

$$
D \rightarrow C:\left[\left[\mathrm{REP}, \mathrm{IP}_{a}, N_{A}\right] K_{X-}\right] K_{D-}, \operatorname{cert}_{x}, \operatorname{cert}_{D}
$$

$C$ validates $D$ 's signature on the received message, removes the signature and certificate, then signs the contents of the message and appends its own certificate before unicasting the REP to $B$.

$$
C \rightarrow B:\left[\left[\mathrm{REP}, \mathrm{IP}_{a}, N_{A}\right] K_{X-}\right] K_{C-}, \operatorname{cert}_{x}, \operatorname{cert}_{C}
$$

Each node checks the nonce and signature of the previous hop as the REP is returned to the source. This avoids attacks where malicious nodes instantiate routes by impersonation and re-play of X's message. When the source receives the REP, it verifies the destination's signature and the nonce returned by the destination.

\section{Route Maintenance}

ARAN is an on-demand protocol. When no traffic has occurred on an existing route for that route's lifetime, the route is simply de-activated in the route table. Data received on an inactive route causes nodes to generate an Error (ERR) message. Nodes also use ERR messages to report links in active routes that are broken due to node movement. All ERR messages must be signed. For a route between source $A$ and destination $X$, a node $C$ generates the ERR message for its neighbor $B$ as follows:

$$
C \rightarrow B:\left[E R R, \mathrm{IP}_{A}, \mathrm{IP}_{X}, N_{c}\right] K_{C-}, \operatorname{cert}_{c}
$$

This message is forwarded along the path toward the source without modification. A nonce ensures that the ERR message is fresh.

It is extremely difficult to detect when ERR messages are fabricated for links that are truly active and not broken. However, the signature on the message prevents impersonation and enables non-repudiation. A node that transmits a large number of ERR messages, whether the ERR messages are valid or fabricated, should be avoided.

\section{E. Responses to Erratic Behavior}

Erratic behavior can come from a malicious node, but it can also come from a friendly node that is malfunctioning. ARAN's response does not differentiate between the two and regards all erratic behavior as the same. Erratic behavior includes the use of invalid certificates, improperly signed messages, and misuse of route error messages. ARAN's response to erratic behavior is a local decision and the details are left to implementors. We discuss how susceptible ARAN is to this behavior in the next section.

\section{F. Potential Optimizations}

Although we have specified the use of public certificates here, it is clear that intermediary nodes ( $B$ and $C$ in our examples) can easily agree upon and exchange session keys using the certificates that authenticate their participation in route creation. Two nodes can easily share a symmetric key generated with their own private key and the public key of the other. A session key can last the duration of their juxtaposition and can be a symmetric key, $K_{B C}$ to reduce processing costs; equivalently, juxtaposed peers can create low-cost hash chains between themselves for authentication of future messages. Using these optimizations would decrease computational overhead and power consumption. However, even if these optimizations are used, we require that sources and destinations must include full public-key signatures for end-to-end route discovery and setup messages.

\section{G. Key Revocation}

In some environments with strict security criteria, the required certificate revocation mechanism must be very reliable 
and expensive. Due to the desired low overhead in wireless networks and the lower standards of security sought in the managed-open and open environments, a best-effort immediate revocation service can be provided that is backed up by the use of limited-time certificates.

In the event that a certificate needs to be revoked, the trusted certificate server, $T$, sends a broadcast message to the ad hoc group that announces the revocation. Calling the revoked certificate cert $_{r}$, the transmission appears as:

$$
T \rightarrow \text { broadcast : }\left[\text { revoke, } \operatorname{cert}_{r}\right] K_{T-}
$$

Any node receiving this message re-broadcasts it to its neighbors. Revocation notices need to be stored until the revoked certificate would have expired normally. Any neighbor of the node with the revoked certificate needs to reform routing as necessary to avoid transmission through the now-untrusted node.

This method is not failsafe. In some cases, the untrusted node that is having its certificate revoked may be the sole connection between two parts of the ad hoc network. In this case, the untrusted node may not forward the notice of revocation for its certificate, resulting in a partition of the network, that lasts until the untrusted node is no longer the sole connection between the two partitions.

At the time that the revoked certificate should have expired, the untrusted node is unable to renew the certificate, and routing across that node ceases. Additionally, to detect this situation and to hasten the propagation of revocation notices, when a node meets a new neighbor, it can exchange a summary of its revocation notices with that neighbor; if these summaries do not match, the actual signed notices can be forwarded and re-broadcasted to restart propagation of the notice.

\section{H. ARAN in Open Environments}

One of the key characteristics of ARAN is that attackers gain little advantage within ARAN by having additional certificates. This makes ARAN well suited for use in open environments where no user is unauthorized to participate in route creation (see Section IV). Open 802.11 networks (often called "rooftop networks") in particular have become widespread: http://www. nodedb. com lists 8,900 open access points around the world.

Open wireless access points running open DHCP can extend their coverage if participating nodes run ARAN. Nodes can register for a DHCP address and then request that a public key they provide is signed by the DHCP/certificate server.

Until now, we have assumed that only authorized nodes can participate in ARAN route creation; however, even participating nodes are prevented from malicious actions such as introducing loops, blackholes, and other attacks covered in Section III. Therefore, ARAN itself does not need to be modified. The remaining risk is that attacking nodes can repeatedly change their MAC addresses and continually ask for new DHCP addresses as well as new certificates. Thus, the open environment does have limitations. In particular, it allows certificate holders to flood the network with data packets. This attack is an option in the managed environment, except that the certificate can be revoked without giving the user the ability to receive renewed authorization.

Jakobsson and Juels have an excellent method of combating this problem: proof of work protocols [33]. To summarize this approach, clients are required to solve a puzzle before a request is satisfied, such as factoring a number. The puzzles could require additional work as resources become more scarce. This increases the resources required of attackers to successfully attack the system proportional to the threat of the attack. Alternatively, certificates can cost money, limiting the ability of the attackers to request them limitlessly. A short lifetime on certificates can also help manage the network.

\section{SeCurity AnAlysis}

In this section, we provide a security analysis of ARAN by evaluating its robustness in the presence of the attacks introduced in Section III. As mentioned earlier, we do not consider denial-of-service attacks based on non-cooperation or aggressive participation, which are possible against all ad hoc routing protocols.

Unauthorized participation: Since all ARAN packets must be signed, a node cannot participate in routing without authorization from the trusted certificate server. This access control therefore rests in the security of the trusted authority, the authorization mechanisms employed by the trusted authority, the strength of the issued certificates, and the revocation mechanism. Although we do not detail these functions explicitly, except for certificate revocation, they have been extensively studied by others.

In practice, many single-hop 802.11 deployments already use VPN certificates; this is the case on the UMass campus. Mechanisms for authenticating users to a trusted certificate authority are numerous; a significant list is provided by Schneier [34]. The trusted authority is also a single point of failure and attack; however, multiple redundant authorities may be used (e.g., as by Zhou and Haas [17]).

Spoofed Route Signaling: Route discovery packets contain the certificate of the source node and are signed with the source's private key. Similarly, reply packets include the destination node's certificate and signature, ensuring that only the destination can respond to route discovery. This prevents impersonation attacks where either the source or destination nodes is spoofed.

Fabricated Routing Messages: Since all routing messages must include the sending node's certificate and signature, ARAN ensures non-repudiation and prevents spoofing and unauthorized participation in routing. ARAN does not prevent fabrication of routing messages, but it does offer a deterrent by ensuring non-repudiation. A node that continues to inject false messages into the network may be excluded from future route computation.

Alteration of Routing Messages: ARAN specifies that all fields of RDP and REP packets remain unchanged between source and destination. Since both packet types are signed by the initiating node, any alterations in transit would be detected, and the altered packet would be subsequently discarded. Repeated instances of altering packets could cause other nodes to 
exclude the errant node from routing, though that possibility is not considered here. Thus, modification attacks are prevented.

Securing Shortest Paths: We believe there is no way to guarantee that one path is shorter than another in terms of hop count. Tunneling attacks, such as the one presented in Section III-A.4, are possible in ARAN as they are in any secure routing protocol. Securing a shortest path cannot be done by any means except by physical metrics such as a timestamp in routing messages. Accordingly, ARAN does not guarantee a shortest path, but offers a quickest path which is chosen by the RDP that reaches the destination first. Malicious nodes could save some processing time by not verifying the previous hop's signature on the RDP packet, thus increasing their chances of being on the quickest route. However such an attack is likely to succeed only if it is executed by multiple malicious nodes on a route, or if a malicious node is already on one of many quick routes to the destination. Malicious nodes also have the opportunity in ARAN to lengthen the measured time of a path by delaying REPs as they propagate, in the worse case by dropping REPs, as well as delaying routing after path instantiation. Finally, malicious nodes using ARAN could also conspire to elongate all routes but one, forcing the source and destination to pick the unaltered route; clearly, a difficult task.

Forwarding Attacks: We have not detailed a specific method of secure forwarding. This could be accomplished using the cryptographic material available to ARAN, but would add overhead to the cost of data transmission. A simple method of protecting data packets would be to use the route reply process to instantiate shared keys between neighbors, and to use that shared key the basis for a pair-wise HMAC. This enforces that only certificate owners can forward data. It does not prevent certificate holders from replay attacks, but in any protocol, authorized participants can just as effectively attack the system by flooding the network with valid data packets for routes they create. End-to-end integrity can be ensured by the shared key derivable from the two peers' public keys.

Denial-of-Service Attacks: Denial-of-service attacks can be conducted by nodes with or without valid ARAN certificates. In the certificateless case, all possible attacks are limited to the attacker's immediate neighbors because unsigned route requests are dropped. There are more severe attacks available at the MAC and physical layer than ARAN provides. Nodes with valid certificates can conduct effective attacks, however, by sending many unnecessary route requests. Because these are broadcast and forwarded across the network, an attacker can cause widespread congestion and power-loss to all nodes in the network. Because it is difficult to infer the node's intent at the network level, it can be hard to differentiate between legitimate and malicious RREQs.

\section{NeTwORK PERFORMANCE}

In this section, we evaluate the performance of ARAN using measurements obtained through both simulation and implementation. Simulations enable us to measure the effectiveness and efficiency of ARAN in reasonably large networks, with and without the presence of malicious nodes. Although simulation is a useful tool for anticipating protocol performance in real networks, it needs to be complemented with protocol implementation in order to obtain a more realistic evaluation of the protocol. With this motivation, we begin this section with a characterization of our ARAN implementation over a threenode network; we then use the quantitative results obtained as input to a simulation of a 50 -node network.

\section{A. ARAN Implementation}

Our open-source implementation of the ARAN protocol, called arand, is publicly available from http://signl. cs.umass.edu/software/arand. It is a user-space routing daemon designed to run on Linux systems with kernel 2.4 or higher. The daemon is written in $\mathrm{C}$ and utilizes the Ad hoc Support Library (ASL) written by Kawadia, Zhang, and Gupta [35]. The ASL and its accompanying Linux kernel module are designed to provide a layer of abstraction that serves as a consistent interface to system functionality required by all ad hoc network protocols. These services include adding and deleting kernel routes as well as notification to the userspace daemon that a route to another host is needed. The library and module also provide functionality to keep track of when routes were last used. This allows routing daemons to delete routes that may no longer exist due to node movement.

The cryptographic functions of arand make use of the OpenSSL library (http://www.openssl.org), which provides functions for general purpose cryptographic tasks such as public and private key encryption/decryption, signing, and certificate management. Each mobile node is issued an X.509 certificate signed by a common Certification Authority. The certification authority and mobile node certificates can be created and managed using the aranca script that is available on the arand project site. All routing related communication between the mobile nodes is done using UDP datagrams. These messages include the message types specified by the ARAN protocol such as RDP, REP, and ERR, as well as signed hello messages that are used by nodes to discover neighbors.

A typical interaction between mobile nodes running arand proceeds as follows. A user on node $A$ attempts to establish a network connection to node $C$. The kernel on $A$ searches its routing table for a route to $C$, but does not find one if $A$ and $C$ are out of signal range and cannot receive each other's hello messages or if a previous route between $A$ and $C$ has expired and been deleted from the kernel routing table. arand is notified of the need for a route to $C$ by the Ad hoc Support Library, which in turn uses the TUN/TAP feature of the Linux kernel. arand running on $A$ checks its state information and determines that it does not have any pending route requests for destination $C$. It then creates a new RDP message signed with its private key and broadcasts this route request on the network. The protocol then follows the steps specified in Section V.

Each node must cryptographically sign and verify each routing message along a path. These cryptographic operations are relatively expensive, especially when compared to other ad hoc routing protocols that do very little computation per message. It is important to note however, that only the routing control messages between nodes are subject to signing/verifying. Data packets exchanged between nodes after a route has been set 


\begin{tabular}{|l|r|}
\hline \hline \multicolumn{1}{|l|}{ Average (ms) \pm Std. Dev. } \\
\hline Laptop: & \\
512 bit RSA key: & $2.2 \pm 0.44$ \\
768 bit RSA key: & $4.3 \pm 0.52$ \\
1024 bit RSA key: & $7.6 \pm 0.62$ \\
\hline iPAQ: \\
512 bit RSA key: & $45.4 \pm 1.14$ \\
768 bit RSA key: & $109.2 \pm 1.64$ \\
1024 bit RSA key: & $199.7 \pm 2.21$ \\
\hline \hline
\end{tabular}

TABLE III

RAW TIME TO PROCESS AN RDP PACKET. LAPTOP: 1200MHZ PENTIUM 3, 512MB RAM. HANDHELD: HP IPAQ 3850 206MHz INTEL STRONG ARM 32-BIT RISC PROCESSOR, 64 MB RAM

up are not processed by arand in any way; they do not have attached certificates and are not signed. Once a route is set up, the routing daemon is out of the picture until that route becomes invalid and is needed again.

\section{B. ARAND Performance}

We have conducted two types of tests to determine the overhead of using certificates and signatures in ARAN. These tests include measurements of raw processing time per routing packet for different key sizes, and measurements of the average route acquisition latency.

We note that the energy cost of cryptographic operations could be of some concern, particularly in resource-constrained mobile devices. However, the energy consumed by wireless communication is significantly higher; a single bit transmission consumes over 1000 times more energy than a single 32-bit computation [36]. Additionally, route discovery is performed infrequently in most ad hoc networks. We therefore do not consider the energy consumption of cryptographic computations to be significant, and do not measure it in our experiments. The wireless communication overhead is quantified in section VII-C.

1) Message Processing Time: We examined the raw processing time expended at a node for an ARAN packet. Specifically, we measured the processing time required for a node to receive an RDP message from a neighbor that is not the initial sender of the RDP, verify that the certificate attached by the neighbor the message was received from is valid, verify the neighbor's signature on the message, strip off the neighbor's certificate, add its own certificate, sign the message, and then rebroadcast the message. We make the distinction between a forwarded RDP and one received from the initial sender because the former is larger since it includes the certificate and signature of the neighbor as well that of the node that originally sent the RDP: both signatures are checked, and our simulations reflect this. Measuring per node processing time on this type of message gives us an upper bound on the processing time for a routing message at each node. Hello messages and error messages (RERR) require less processing time.

We conducted this test by mirroring the sequence of function calls that are performed when an RDP message is received by arand; however, we do not consider the time spent performing operations on the state that is maintained in arand (such as looking through a list of RDPs to determine whether we have already seen a particular message). This simplified the test and allowed us to focus on the time spent on the cryptographic operations instead of state maintenance, which is negligible in comparison.

The main purpose of this performance test was to illustrate the expense of processing routing messages with two different types of devices that are likely to participate in an ad hoc network using the ARAN protocol. Table III shows our results. We measured processing time for both a laptop and a handheld computer over three different RSA key sizes: 512,768 , and 1024 bits. For both devices, increasing the key size by 256 bits roughly doubles processing time. Perhaps most striking in Table III is the difference in processing times between the laptop and the handheld. For each key size, the processing time is between 20 and 30 times slower on the handheld than on the laptop. From this, it is clear that the processing power of the nodes expected to participate in an ad hoc network can limit key sizes if routing overhead is a limiting factor. In other words, ARAN is not appropriate for low-resource devices when node mobility is high and route changes are very frequent.

2) Route Acquisition Latency: We also measured the average route acquisition latency, which is the delay from route request initiation to the receipt of a corresponding reply. The results of measuring latency in this way depend on the number and topology of network nodes. For simplicity, and because creating an elaborate topology with actual machines would be unwieldy, we created a simple topology with three nodes oriented in a straight line topology: $A \leftrightarrow B \leftrightarrow C$. The node in the middle is within range of the two end nodes, but the end nodes are not in range of each other. We measured the route acquisition latency for a route request from $A$ to $C$. All routing messages are sent through the intermediate node, $B$.

For comparison, we have executed this experiment for both AODV using the AODV-UIUC version 0.5 (http: // aslib.sourceforge.net), which is an AODV daemon written to use the Ad hoc Support Library, and for ARAN using arand version 0.3.2. All nodes running arand are using version 0.9.6d of the OpenSSL library. Both routing daemons were modified to record the time when a route request is sent and when its corresponding route reply is received. Also, when a route reply is received, we disabled the actual addition of the newly discovered route to the kernel to allow us to continually request routes without restarting the daemon. On the sender node $A$, we ran a script that automatically generated network traffic for destination $C$, causing the daemon to request a route. The script then sleeps for a random number of seconds before generating traffic again. We measure route latency across three different RSA key sizes used by the nodes. However, in each case the CA signed client certificates were signed with a 1024bit RSA key.

We measured average route acquisition latency using two topologies consisting of different types of devices. Table IV shows average route acquisition latency for the topology where node $A$ was a laptop, and nodes $B$ and $C$ were iPAQs. The 


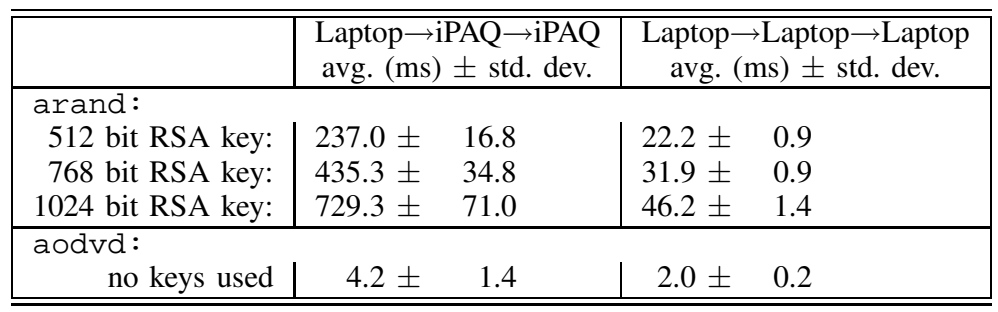

TABLE IV

THE AVERAGE LATENCY TO ACQUIRE A ROUTE IN TWO NETWORKS: FROM A LAPTOP THROUGH TWO IPAQS, AND THROUGH A ROUTE CONSISTING OF THREE LAPTOPS.

last column of Table IV shows the average route acquisition latency for the topology where nodes $A, B$, and $C$ are all Pentium 3 laptops. As can be seen from Table IV, in the iPAQ topology, the route acquisition latency using arand is between 56 and 175 times slower than aodvd, depending on the size of the key used on the nodes running arand. When laptops, which have significantly greater processing power than the iPAQs, are used, arand is only between 11 and 23 times slower than aodvd, depending on the size of the key. Even with the comparatively high route acquisition latencies experienced with the iPAQ topology, these latencies are still rather small compared with the duration of the typical connection between nodes in an ad hoc network. It is important to note that after a route is set up between two nodes, data packets exchanged between the nodes do not involve the ARAN routing daemon in any way, so this cost is incurred only once unless the route breaks. The lifetime of a route between a pair of nodes will typically be much longer than the time necessary for route acquisition in all ad hoc networks, except those with the most rapidly changing topologies. The initially higher cost to acquire a route will likely turn out to be an acceptable price to pay to ensure node authentication and prevention of modified or forged routing messages.

\section{Simulated Network Performance}

We performed our evaluations using the Global Mobile Information Systems Simulation Library (GloMoSim) [37]. We used an IEEE 802.11 MAC layer and CBR traffic over UDP.

We simulated two types of field configurations: 20 nodes distributed over a $670 \mathrm{~m} \times 670 \mathrm{~m}$ terrain, and 50 nodes over a $1000 \mathrm{~m} \times 1000 \mathrm{~m}$ terrain. The initial positions of the nodes were random. Node mobility was simulated according to the random waypoint mobility model. The node transmission range was $250 \mathrm{~m}$. We ran simulations for constant node speeds of $0,1,5$ and $10 \mathrm{~m} / \mathrm{s}$, with pause time fixed at 30 seconds. We simulated five CBR sessions in each run, with random source and destination pairs. Each session generated 1000 data packets of 512 bytes each at the rate of 4 packets per second.

ARAN was simulated using a 512 bit key and 16 byte signature. These values are reasonable to prevent compromise during the short time nodes spend away from the certificate authority and in the ad hoc network.
For both ARAN and AODV, we assumed a routing packet processing delay of $1 \mathrm{~ms}$. This value was obtained through field testing of the AODV protocol implementation [38]. An additional processing delay of $2.2 \mathrm{~ms}$ was added for ARAN to account for the cryptographic operations. This value was obtained through the implementation testing of ARAN, as reported in table III. Additionally, a random delay between 0 and $10 \mathrm{~ms}$ was introduced before the transmission of a broadcast packet in order to minimize collisions. This is required since the 802.11 MAC protocol does not perform an RTS/CTS exchange for broadcast packets. Since we are working with fairly dense networks, the probability of collision of broadcast packets becomes quite high in the absence of this random delay.

In order to compare the performance of ARAN and AODV, both protocols were run under identical mobility and traffic scenarios. A basic version of AODV was used, which did not include optimizations such as the expanding ring search and local repair of routes. This enables a consistent comparison of results.

We evaluated six performance metrics:

(1) Packet Delivery Fraction: This is the fraction of the data packets generated by the CBR sources that are delivered to the destination. This evaluates the ability of the protocol to discover routes.

(2) Routing Load (bytes): This is the ratio of overhead bytes to delivered data bytes. The transmission of a control byte at each hop along the route was counted as one transmission in the calculation of this metric. ARAN suffers from larger control overhead due to certificates and signatures stored in packets. Notice that many other secure routing protocols assume the existence of key information without accounting for the costs of distributing it; while ARAN may appear more expensive it is in part because our analysis is more complete.

(3) Routing Load (packets): Similar to the above metric, but a ratio of control packet overhead to data packet overhead.

(4) Average Path Length: This is the average length of the paths discovered by the protocol. It was calculated by averaging the number of hops taken by each data packet to reach the destination.

(5) Average Route Acquisition Latency: This is the average delay between the sending of a route request/discovery packet by a source for discovering a route to a destination and the receipt of the first corresponding route reply. If a 


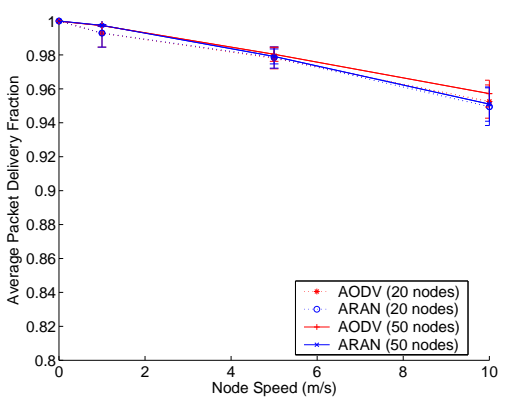

(a) Packet Delivery Fraction.

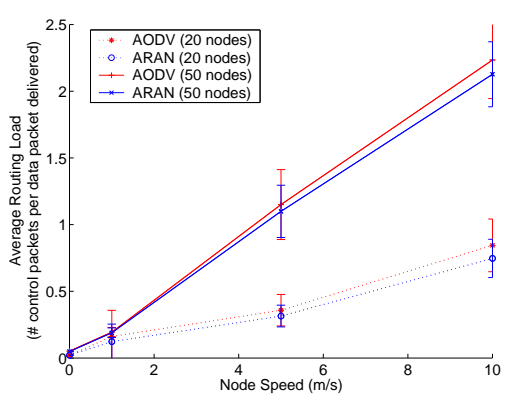

(d) Routing Load (packets).

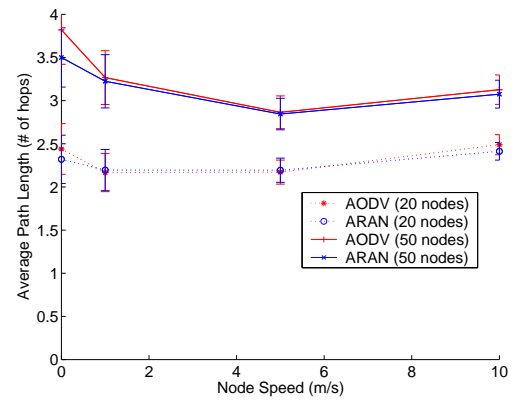

(b) Average Path Length.

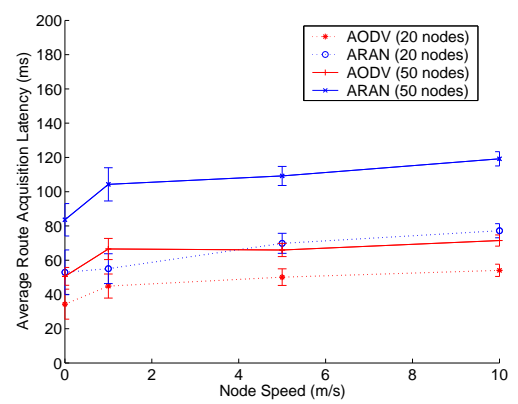

(e) Route Acquisition Delay.

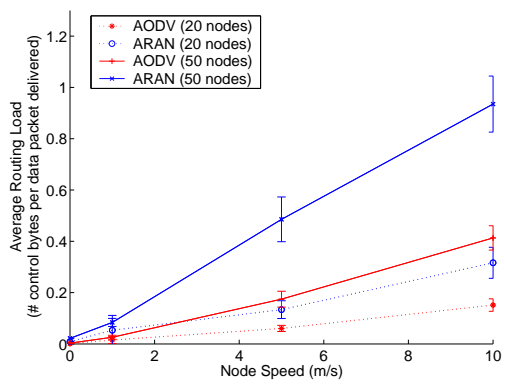

(c) Routing Load (bytes).

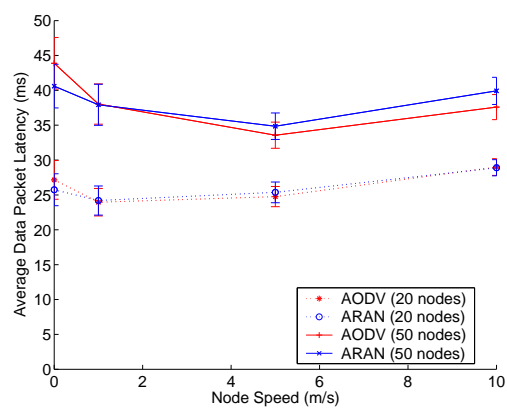

(f) End-to-End Delay of Data Packets.

Fig. 1. Simulation Results.

route request timed out and needed to be retransmitted, the sending time of the first transmission was used for calculating the latency.

(6) Average End-to-End Delay of Data Packets: This is the average delay between the sending of the data packet by the CBR source and its receipt at the corresponding CBR receiver. This includes all the delays caused during route acquisition, buffering and processing at intermediate nodes, and retransmission delays at the MAC layer.

1) Performance Results: Figures 1(a) through 1(f) show the observed results for both the 20 and 50 node networks. Each data point is an average of 10 simulation runs with identical configuration but different randomly generated mobility patterns. Error bars report 95\% confidence intervals and are small in all cases.

As shown in Figure 1(a), the packet delivery fraction obtained using ARAN is $95 \%$ or higher in all scenarios and almost identical to that obtained using AODV. This suggests that ARAN is highly effective in discovering and maintaining routes for delivery of data packets, even with relatively high node mobility.

Traditionally, the shortest path to a destination (in terms of number of hops) is considered to be the best routing path. AODV explicitly seeks shortest paths using the hop count field in the route request/reply packets. ARAN, on the other hand, assumes that the first route discovery packet to reach the destination must have traveled along the best path (i.e., the path with the least congestion).
The average path length graphs are almost identical for the two protocols, as shown in Figure 1(b). This indicates that even though ARAN does not explicitly seek shortest paths, the first route discovery packet to reach the destination usually travels along the shortest path. Hence ARAN is as effective in finding the shortest path as AODV. It should be noted, however, that in networks with significantly heavier data traffic loads, congestion could prevent the discovery of the shortest path with ARAN.

Figs. 1(c) and 1(d) show the routing load measurements in bytes and packets, respectively. ARAN's byte routing load is significantly higher and increases to nearly $94 \%$ for 50 nodes moving at $10 \mathrm{~m} / \mathrm{s}$, as compared to $42 \%$ for AODV. This is due to the security data. However, the number of control packets transmitted by the two protocols is roughly equivalent. Figure 1(d) shows the average number of control packets transmitted per delivered data packet. AODV has the advantage of smaller control packets; smaller packets have a higher probability of successful reception at the destination. However, due to the IEEE 802.11 MAC layer overhead for unicast transmissions, a significant part of the overhead of control packets is in acquiring the channel. In this respect, the two protocols demonstrate nearly the same amount of packet overhead.

Figure 1(e) shows that the average route acquisition latency for ARAN is approximately double that for AODV. While processing ARAN control packets, each node has to verify the digital signature of the previous node, and then replace 


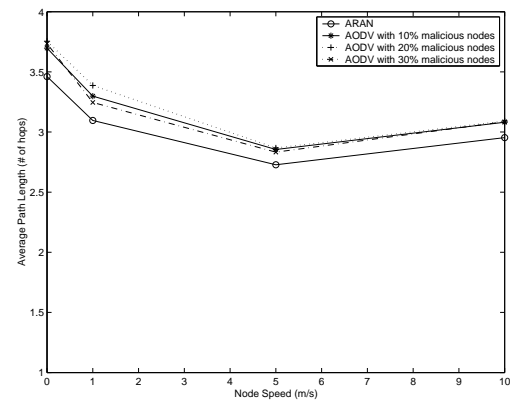

(a) Average path length.

Fig. 2. Effect of malicious node behavior.

this with its own digital signature, in addition to the normal processing of the packet as done by AODV. The cryptographic operations cause additional delays at each hop, and so the route acquisition latency increases.

We found through our implementation testing that the route acquisition latency using ARAN is 11 to 23 times higher than that using AODV, as reported in table IV. On the other hand, our simulations show that it is less than twice as high, as shown in figure 1(e). The reason for this discrepancy is the random delay we introduced before transmitting broadcast packets in the simulations, as described in section VII-C. Since the network used in the implementation testing is simple and not dense, the random delay was not required there. However, it is necessary in the relatively dense simulated networks for reducing collisions.

The data packet latencies for the two protocols are again almost identical as shown in Figure 1(f). Although ARAN has a higher route acquisition latency, the number of route discoveries performed is a small fraction of the number of data packets delivered. Hence the effect of the route acquisition latency on average end-to-end delay of data packets is not significant. The processing of data packets is identical when using either protocol, and so the average latency is nearly the same.

2) Effect of Malicious Node Behavior: The experiments described in the previous sections compare the performance of ARAN and AODV when all the nodes in the network are well-behaved or benign. We conducted additional experiments to determine the effect of malicious node behavior on the two protocols. We used a field configuration of 50 nodes distributed over a $1000 \mathrm{~m} \times 1000 \mathrm{~m}$ area.

As illustrated earlier, various types of malicious behavior are possible when using AODV. The malicious behavior simulated in these experiments is as follows: whenever a malicious node forwards an RREQ or RREP packet, it illegally resets the hop count field to 0 , thus pretending to be only one hop away from the source or destination node, respectively. The objective of a malicious node is to try to force the selected routes to pass through itself by exploiting the routing protocol, so that it is able to overhear and potentially modify or drop data packets.

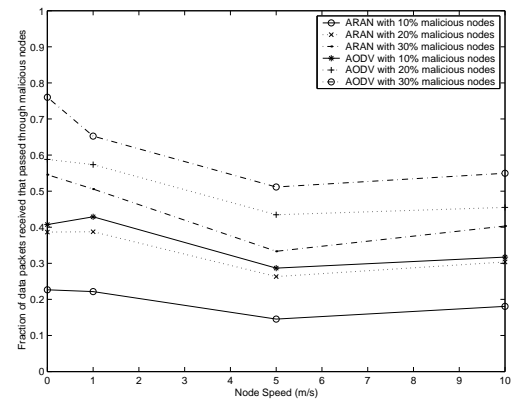

(b) Fraction of data packets received that passed through malicious nodes.
The effect of this behavior is that non-shortest paths containing malicious nodes are likely to be selected, and the average path length increases. ARAN, on the other hand, cannot be exploited in this fashion. When using ARAN, the selected route could still pass through a malicious node; however, the routing protocol cannot be manipulated to force this behavior.

We ran simulations with $10 \%, 20 \%$ and $30 \%$ malicious nodes for each protocol. The malicious nodes were selected randomly. We measured the following metrics:

Average path length: Malicious nodes can exploit AODV so that non-shortest paths are selected, while such exploitation is not possible with ARAN. This metric indicates the extent of path elongation in AODV in the presence of different percentages of malicious nodes. The metric is important because longer routes result in greater routing overhead and longer data packet delays.

Fraction of data packets received that passed through malicious nodes: This metric indicates the fraction of data packets that traverse malicious nodes when using each routing protocol, in the presence of different percentages of malicious nodes. The metric is important because data packets passing through malicious nodes are overheard by these nodes, and could potentially be modified or dropped.

Figure 2 illustrates the results of the experiments. As seen in Figure 2a, the average path length increases about $10 \%$ for AODV in the presence of malicious nodes. Figure $2 b$ shows that when using AODV, a much larger fraction of data packets passes through malicious nodes, as compared to using ARAN. For instance, in the presence of $10 \%$ malicious nodes with no node mobility, only $22 \%$ of data packets pass through malicious nodes when using ARAN, as compared to almost $40 \%$ when using AODV. This is because malicious nodes can potentially manipulate AODV to make routes pass through themselves.

\section{Energy Costs}

ARAN's energy expenditure is high in comparison to protocols that employ hash chains, like Ariadne. This is because ARAN spends longer time verifying signatures. These costs 
must be viewed in context of other energy costs of the handheld device. It is important to realize that in an ad hoc network, the handheld device must be powered at all times for successful reception of route requests. The question we must ask is, what is the additional energy spent during ARAN's cryptographic operations?

The largest energy drain on a handheld device is due to operating a wireless network interface card (NIC), as several researchers have found. From our experiments (see Table 3 in the paper), we know the running time for an iPAQ to process an RDP packet is $45 \mathrm{~ms}$. Many measurement studies exist on our equipment. Using values record by Kremer et al [5], Bahl et al [2], and Cho [4] as a baseline, we can provide a estimate of the costs of ARAN's cryptographic operation.

If we set the CPU power cost as $12 \%$ of $1250 \mathrm{~mW}$ as per Kremer's measurements, then the energy usage for processing an RDP packet is $150 \mathrm{~mW} \cdot 0.045 \mathrm{sec}=6.8 \mathrm{~mJ}$. Costs equal to ARAN's CPU operations will be spent by an idle radio (805mW [2]) coupled with an idle iPAQ (470mW [4]) every $5 \mathrm{~ms}$.

\section{CONCLUSION}

Popular ad hoc routing protocols are subject to a variety of attacks, which, through modification or fabrication of routing messages or impersonation of other nodes, can allow attackers to influence a victim's selection of routes or enable denial-ofservice attacks. We have shown a number of such attacks, and how they are easily exploited in two ad hoc routing protocols under consideration by the IETF.

Our protocol, ARAN, provides secure routing for the managed-open and open environments. ARAN provides authentication and non-repudiation services using cryptographic certificates that guarantees end-to-end authentication. In doing so, ARAN limits or prevents attacks that can afflict other insecure protocols. ARAN is a simple protocol that does not require significant additional work from nodes within the group. Our simulations and experiments show that ARAN is as effective as AODV in discovering and maintaining routes. The cost of ARAN is larger routing packets, which result in a higher overall routing load, and higher latency in route discovery because of the cryptographic computation that must occur.

\section{REFERENCES}

[1] S. Savage, N. Cardwell, D. Wetherall, and T. Anderson, "TCP Congestion Contorl with a Misbehaving Receiver," Computer Communication Review, vol. 29, no. 5, 1999.

[2] E. Shih, P. Bahl, and M. Sinclair, "Wake on Wireless: An Event Driven Energy Saving Strategy for Battery Operated Devices," in Proc. MobiCom, 2002.

[3] M. Stemm and R. H. Katz, "Measuring and Reducing Energy Consumption of Network Interfaces in Handheld Devices," IEEE Transactions on Communications, vol. E80-B, no. 8, pp. 1125-1131, 1997.

[4] S. Cho, "Power Management of the iPAQ," USC/ISI Technical Report, http://pads.east.isi.edu/presentations/misc/ sjcho-pm-report.pdf.

[5] U. Kremer, J. Hicks, and J. Rehg, "A Compilation Framework for Power and Energy Management on Mobile Computers," in Proc. Workshop on Parallel Computing (LCPC), Aug. 2001.

[6] Y. Hu, A. Perrig, and D. Johnson, "Ariadne: A Secure On-Demand Routing Protocol for Ad Hoc Networks," in Proc. MobiCom, Sep. 2002.

[7] R. Canetti, D. Song, A. Perrig, and D. Tygar, "Efficient and Secure Source Authentication for Multicast," in Proc. ISOC NDSS, Feb. 2001.
[8] K. Sanzgiri, B. Dahill, B. N. Levine, C. Shields, and E. M. BeldingRoyer, "A Secure Protocol for Ad hoc Networks," in Proc. ICNP, Nov. 2002.

[9] C. E. Perkins and E. M. Royer, "Ad hoc On-Demand Distance Vector Routing," in Proc. WMCSA, Feb. 1999.

[10] D. Johnson, D. Maltz, and Y.-C. Hu, "The Dynamic Source Routing Protocol for Mobile Ad Hoc Networks (DSR)," IEEE Internet Draft, Apr. 2003.

[11] S. Murthy and J. Garcia-Lunca-Aceves, "An Efficient Routing Protocol for Wireless Networks," ACM Mobile Networks and Applications Journal, Oct. 1996.

[12] V. Park and M. Corson, "A Highly Adaptive Distributed Routing Algorithm for Mobile Wireless Networks," in Proc. Infocom, Apr. 1997.

[13] C. E. Perkins and P. Bhagwat, "Highly Dynamic Destination-Sequenced Distance-Vector Routing (DSDV) for Mobile Computers," Computer Communications Review, Oct. 1994.

[14] J.-P. HuBaux, L. Buttyan, and S. Capkun, "The Quest for Security in Mobile Ad Hoc Networks," in Proc. MobiCom, Oct. 2001.

[15] S. Capkun, L. Buttyan, and J.-P. Hubaux, "Self-Organized Public-Key Management for Mobile Ad hoc Networks," IEEE Transactions on Mobile Computing, vol. 2, no. 1, Jan.-Mar. 2003.

[16] J. Kong, P. Zerfos, H. Luo, S. Lu, and L. Zhang, "Providing Robust and Ubiquitous Security Support for Wireless Mobile Networks," in Proc. ICNP, Nov. 2001.

[17] L. Zhou and Z. J. Haas, "Securing Ad Hoc Networks," IEEE Network, vol. 13, no. 6, pp. 24-30, 1999.

[18] S. Buchegger and J.-Y. Le Boudec, "Performance Analysis of the CONFIDANT Protocol (Cooperation Of Nodes: Fairness In Dynamic Ad-hoc NetTworks)," in Proc. MobiCom, Sep. 2002.

[19] S. Marti, T. Giuli, K. Lai, and M. Baker, "Mitigating Routing Misbehavior in Mobile Ad Hoc Networks," in Proc. MobiCom, Aug. 2000.

[20] M. Just, E. Kranakis, and T. Wan, "Resisting Malicious Packet Dropping in Wireless Ad Hoc Networks," in Proc. ADHOC-NOW, Oct. 2003.

[21] L. Buttyan and J. Hubaux, "Enforcing Service Availability in Mobile Ad-Hoc WANs," in Proc. MobiHoc, Aug. 2000.

[22] S. Sundaramurthy and E. Belding-Royer, "The AD-MIX Protocol for Encouraging Participation in Mobile Ad hoc Networks," in Proc. IEEE ICNP, Nov. 2003.

[23] P. Papadimitratos and Z. Haas, "Secure Message Transmission in Mobile Ad hoc Networks," in Proc. ACM WiSe, Sep. 2003.

[24] Y. Hu, D. Johnson, and A. Perrig, "SEAD: Secure Efficient Distance Vector Routing for Mobile Wireless Ad Hoc Networks," in Proc. WMCSA, Jun. 2002

[25] M. G. Zapata and N. Asokan, "Securing Ad hoc Routing Protocols," in Proc. ACM WiSe, Sep. 2002.

[26] S. Yi, P. Naldurg, and R. Kravets, "Security-Aware Ad Hoc Routing for Wireless Networks," in Proc. MobiHoc, Oct. 2001.

[27] P. Papadimitratos and Z. Haas, "Secure Routing for Mobile Ad hoc Networks," in Proc. SCS CNDS, Jan. 2002.

[28] C. Karlof and D. Wagner, "Secure Routing in Wireless Sensor Networks: Attacks and Countermeasures," in Proc. IEEE SNPA, May 2003.

[29] Y. Hu, A. Perrig, and D. Johnson, "Packet Leashes: A Defense against Wormhole Attacks in Wireless Networks," in Proc. Infocom, Apr. 2003.

[30] B. Awerbuch, D. Holmer, and H. Rubens, "Provably Secure Competitive Routing against Proactive Byzantine Adversaries via Reinforcement Learning," John Hopkins University, Tech. Rep., May 2003.

[31] Y. Zhang and W. Lee, "Intrusion Detection in Wireless Ad-Hoc Networks," in Proc. MobiCom, Aug. 2000.

[32] S. Gwalani, K. Srinivasan, G. Vigna, E. Belding-Royer, and R. Kemmerer, "An Intrusion Detection Tool for AODV-based Ad hoc Wireless Networks," in Proc. ACSAC, Dec. 2004.

[33] M. Jakobsson and A. Juels, "Proofs of Work and Breadpudding Protocols," in Proc. Communications and Multimedia Security, Sep. 1999.

[34] B. Schneier, Applied Cryptography, 2nd ed. John Wiley \& Sons, Inc., New York, 1996.

[35] V. Kawadia, Y. Zhang, and B. Gupta, "System Services for Implementing Ad-Hoc Routing: Architecture, Implementation and Experiences," in Proc. MobiSys, May 2003.

[36] K. Barr and K. Asanovic, "Energy Aware Lossless Data Compression," in Proc. MobiSys, May 2003.

[37] L. Bajaj, M. Takai, R. Ahuja, K. Tang, R. Bagrodia, and M. Gerla, "GlomoSim: A Scalable Network Simulation Environment," UCLA, Tech. Rep. CSD Technical Report \#990027, 1997.

[38] I. D. Chakeres and E. M. Belding-Royer, "The Utility of Hello Messages for Determining Link Connectivity," in Proc. WPMC, Oct. 2002. 